\title{
Approximation of convex bodies by inscribed simplices of maximum volume
}

\author{
Marek Lassak
}

Received: 19 March 2010 / Published online: 3 May 2011

(C) The Author(s) 2011. This article is published with open access at Springerlink.com

\begin{abstract}
The Banach-Mazur distance between an arbitrary convex body and a simplex in Euclidean $n$-space $E^{n}$ is at most $n+2$. We obtain this estimate as an immediate consequence of our theorem which says that for an arbitrary convex body $C$ in $E^{n}$ and for any simplex $S$ of maximum volume contained in $C$ the homothetical copy of $S$ with ratio $n+2$ and center in the barycenter of $S$ contains $C$. In general, this ratio cannot be improved, as it follows from the example of any double-cone.
\end{abstract}

Keywords Approximation - Banach-Mazur distance $\cdot$ Convex body $\cdot$ Double-cone . Simplex · Volume

\section{Mathematics Subject Classification (2000) $\quad 52 \mathrm{~A} 21 \cdot 52 \mathrm{~A} 10 \cdot 46 \mathrm{~B} 20$}

Denote by $\mathcal{C}^{n}$ the family of all convex bodies and by $\mathcal{M}^{n}$ the family of all centrally symmetric convex bodies in Euclidean $n$-dimensional space $E^{n}$.

Finding simplices of large volume in convex bodies has a long history. See, for instance, the papers by Blaschke (1917) on the maximum area of triangles in planar convex bodies, by MacKinney McKinney (1974) on simplices of maximum volume in centrally symmetric convex bodies, and the survey article Hudelson et al. (1996) by Hudelson, Klee and Larman on simplices of large volume in cubes.

Let $A$ be an $(n-1)$-dimensional convex body and let $I=a b$ be a perpendicular to the hyperplane containing $A$ non-degenerated segment centered at a point of $A$. We call the convex hull conv $(A \cup I)$ the double pyramid with base $A$ and apices a and $b$. If $B$ is a ball of $E^{n-1}$ in place of $A$ and if the centers of $B$ and $I$ coincide, the double-pyramid is called a double-cone.

M. Lassak (凶)

Institute of Mathematics and Physics, University of Technology, 85-789 Bydgoszcz, Poland e-mail: lassak@utp.edu.pl 
Theorem For arbitrary $C \in \mathcal{C}^{n}$ and for any simplex $S$ of maximum volume contained in $C$ we have $C \subset(n+2) S$. For $n \geq 2$ the ratio $n+2$ is best possible and, in particular, it cannot be lessened for every double-cone in part of $C$.

For the proof of the Theorem we need Lemmas 1 and 2 presented below. We give the proof of the Theorem after them.

The first lemma is very easy to show, see the proof of Proposition in Lassak (2006).

Lemma 1 Let $C \in \mathcal{C}^{n}$, and let $S$ be a simplex of maximum volume contained in $C$. Then $S$ is inscribed in $C$ and for every vertex $v$ of $S$ the hyperplane through $v$ parallel to the opposite facet of $S$ supports $C$.

This lemma immediately implies the well known property that the only simplices of maximum volume contained in a ball are the regular inscribed simplices. We will need this fact in the proof of Lemma 2.

Lemma 2 A simplex $S$ contained in a double-cone $D \subset E^{n}$, where $n \geq 3$, with base $B$ is of maximum possible volume if and only if a vertex of $S$ is at an apex of $D$ and the remaining vertices of $S$ are vertices of a regular $(n-1)$-dimensional simplex inscribed in B. For $n=2$ only the "if" part holds true.

Proof By Lemma 1 every simplex of maximum volume contained in $D$ is inscribed in $D$.

I. Arbitrary simplex $P$ inscribed in $D$ with two vertices at two apices of $D$ is not a simplex of maximum volume in $D$.

Let $v_{0}$ be a vertex of $P$ at an apex of $D$, and denote the remaining vertices of $P$ by $v_{1}, \ldots, v_{n}$ (see Fig. 1). Denote by $G$ the hyperplane containing them. Support $D$ by the hyperplane $H$ parallel to $G$ such that $S$ is contained in the strip between $G$ and $H$. Since a vertex from amongst $v_{1}, \ldots, v_{n}$ is the other apex of $D$, we see that $H$ supports $D$ at a point $w$ of the sphere bounding $B$ and that this is the only point of support. Consequently, the distance of $w$ from $G$ is greater than the distance of $v_{0}$ from $G$. Thus the simplex with vertices $v_{1}, \ldots, v_{n}$ and $w$ has volume greater than the volume of $P$. Hence $P$ is not a simplex of maximum volume in $D$.

II. Consider all simplices with a vertex at an apex of $D$ and with the remaining vertices in the boundary of $B$. A simplex from this family has the greatest volume amongst simplices from this family if and only if its vertices lying in the boundary of $B$ are vertices of an $(n-1)$-dimensional regular simplex.

This follows from the $(n-1)$-dimensional version of the property presented immediately after Lemma 1.

III. Every simplex inscribed in D which is not of the form considered in Parts I and II is not a simplex of maximum volume contained in $D$.

Assume the opposite: there exists a simplex $Q_{1}$ not of the form considered in Parts I and II, which is a simplex of maximum volume contained in $D$.

From the assumptions of Parts I and II we conclude that $Q_{1}$ has a vertex $x_{1}$ which is not in $B$ and not in an apex of $D$ (see Fig. 2). Clearly, $x_{1}$ belongs to a segment $a b_{1}$, where $a$ is an apex of $D$ and $b_{1}$ is a boundary point of $B$. There is a unique hyperplane $V_{1}$ supporting $D$ at $x_{1}$. By this uniqueness and by the second statement of Lemma 1 , 


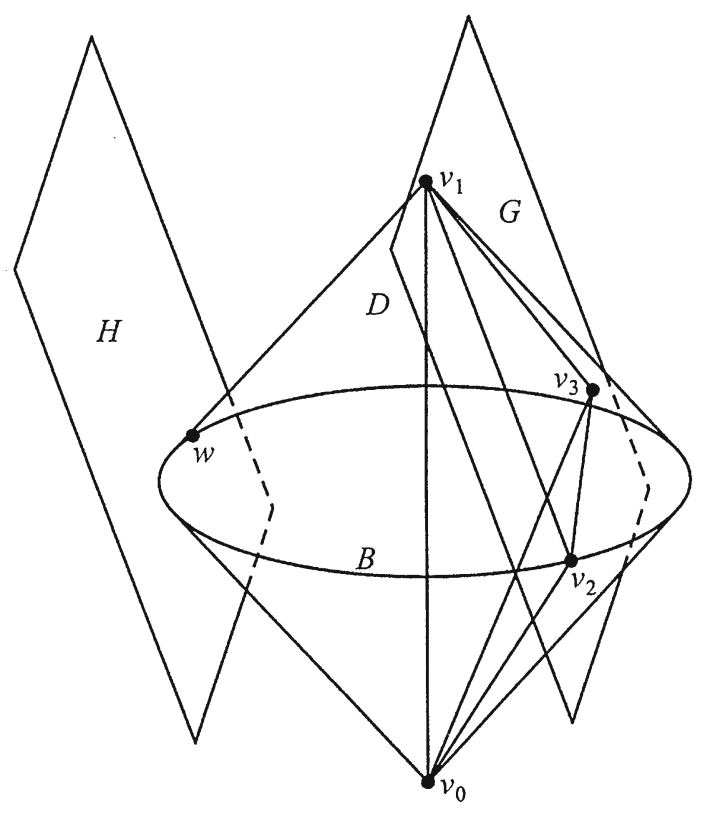

Fig. 1 Illustration to Part I of the proof of Lemma 2

the facet $F_{1}$ of $S$ opposite to $x_{1}$ is parallel to $V_{1}$. Denote by $H_{1}$ the carrying hyperplane of $F_{1}$. Since $x_{1}, b_{1} \in V_{1}$, the distances from $x_{1}$ and $b_{1}$ to $H_{1}$ are equal. Hence the simplex $Q_{2}$ obtained by exchanging $x_{1}$ into $b_{1}$ has the same volume as $Q_{1}$. If there exists a vertex $x_{2}$ of $Q_{2}$ which is not in $B$ and not at an apex of $D$, by analogous procedure we can exchange it by a point $b_{2}$ from the boundary of $B$ obtaining a new simplex $Q_{3}$ of equal volume. Then we repeat this procedure, as long as it is possible. At the last $k$-th stage, from a simplex $Q_{k-1}$ we get a simplex $Q_{k}$ with $n$ vertices in the boundary of $B$. Clearly, one vertex of $Q_{k}$ must be at an apex of $D$ since in the opposite case $Q_{k}$ would not be of maximum volume.

The maximality of the volume of $Q_{k}$ together with Part II imply that the convex hull of its $n$ vertices which are in the boundary of $B$ is a regular $(n-1)$-dimensional simplex inscribed in $B$. This determines the shape of $Q_{k}$.

Our procedure implies that the volumes of $Q_{k-1}$ and $Q_{k}$ are equal. On the other hand this is not true since from the established above shape of $Q_{k}$ we see that its vertex $b_{k-1}$ is farther than the point $x_{k-1}$ from the hyperplane $H_{k-1}$ containing the vertices of $Q_{k}$ different from $b_{k-1}$ (the shape of $Q_{k}$ shows that the parallel hyperplane to $H_{k-1}$ through $b_{k-1}$ supports $D$ only at this point). A contradiction.

IV. From Parts I-III we conclude the thesis of Lemma 2 for $n \geq 3$. An easy proof of the thesis for $n=2$ is left to the reader as an exercise.

The homothetical image of a simplex $S$ with ratio $\lambda>0$ and the center in the barycenter of $S$ is denoted by $\lambda S$. 


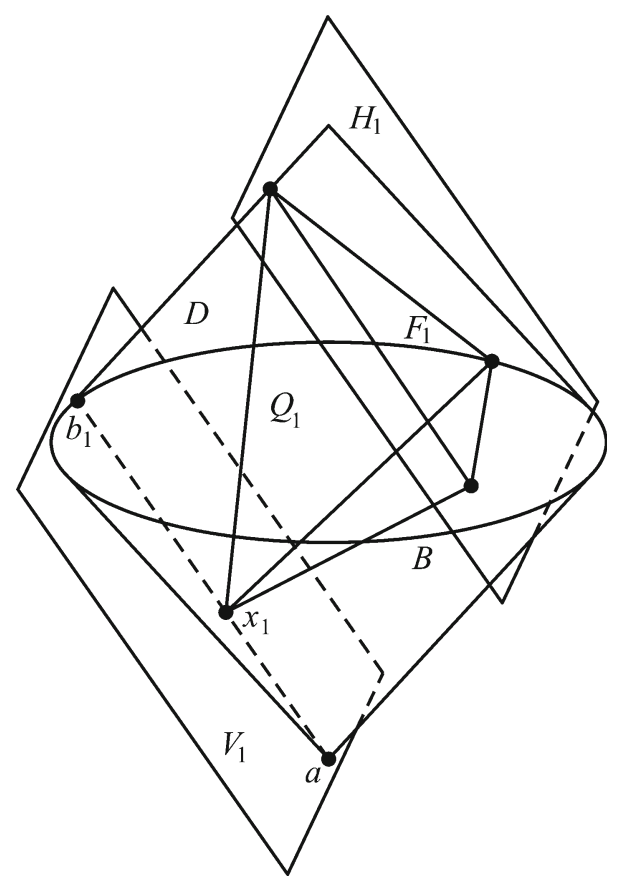

Fig. 2 Illustration to Part III of the proof of Lemma 2

Proof of the Theorem. Denote by $v_{0}, \ldots, v_{n}$ the vertices and by $c$ the barycenter of $S$. Let $P_{i}$ be the hyperplane containing all the vertices of $S$ different from $v_{i}$. We provide the parallel hyperplane $O_{i}$ through $v_{i}$ and the parallel hyperplane $R_{i}$ such that $P_{i}$ is in equal distances from $O_{i}$ and $R_{i}$. Thus, if a point $p$ of $C$ is on the opposite side of $R_{i}$ than $S$, the simplex $S_{i}$ with one vertex at $p$ and with the remaining vertices at the vertices of $S$ different from $v_{i}$ has volume greater than the volume of $S$. This is impossible since $S_{i} \subset C$ and since $S$ is a simplex of maximum volume in $C$. Consequently, every point of $C$ is in the half-space $T_{i}$ bounded by $R_{i}$ which contains $S$. So $C$ is contained in the simplex being the intersection of the half-spaces $T_{0}, \ldots, T_{n}$. Since $c$ is $n+2$ times farther from $R_{i}$ than from $P_{i}$, this simplex is nothing else but $(n+2) S$. We see that $C \subset(n+2) S$.

Now we show that the ratio $n+2$ cannot be diminished for every double-cone $D$. Let us stay with the notation of the first part of this proof. If $n \geq 3$, the position of every simplex $S$ of maximum volume in $D$ is described in Lemma 2, and for $n=2$ take a triangle $S$ being the convex hull of any three vertices of the parallelogram $D$. So one vertex of $S$, say $v_{0}$, is an apex of $D$, and the convex hull of the remaining $n$ vertices is a regular $(n-1)$-dimensional simplex inscribed in the base $B$ of $D$. Of course, $B \subset P_{0}$. Since the other apex of $D$ is in the hyperplane $R_{0}$, the ratio $n+2$ cannot be lessened.

Denote by $D_{s}$ the double-pyramid whose base is a regular $(n-1)$-dimensional simplex inscribed in $B$ and whose apices are the apices of $D$. The estimate $n+2$ in the 
Theorem cannot be lessened also for every convex body $K$ such that $D_{S} \subset K \subset D$. This is an obvious consequence of the second statement of this theorem.

The second aim of this short note is the below presented Corollary on the BanachMazur distance between an arbitrary convex body and a simplex.

Recall that the Banach-Mazur distance of bodies $C_{1}, C_{2} \in \mathcal{C}^{n}$ is the number

$$
\delta\left(C_{1}, C_{2}\right)=\inf _{t, h_{\lambda}}\left\{\lambda ; \quad t\left(C_{2}\right) \subset C_{1} \subset h_{\lambda}\left(t\left(C_{2}\right)\right)\right\}
$$

where $t$ stands for an affine transformation, and $h_{\lambda}$ stands for any homothety with a positive ratio $\lambda$. For more than 70 years this notion with $C_{1}, C_{2} \in \mathcal{M}^{n}$ plays an important role in functional analysis. A survey of results is given by Tomczak-Jaegermann Tomczak-Jaegermann (1989). In the last 20 years also the general situation, when the convex bodies $C_{1}$ and $C_{2}$ are not necessarily centrally symmetric, is considered in many papers. For a larger context see the survey article Szarek (2006) by Szarek. From the celebrated theorem of John John (1948) that $\delta(C, B) \leq n$ for every convex body $C \in \mathcal{C}^{n}$ and the ball $B \subset E^{n}$ we immediately conclude that $\delta\left(C_{1}, C_{2}\right) \leq n^{2}$ for arbitrary $C_{1}, C_{2} \in \mathcal{C}^{n}$. For instance, Rudelson Rudelson (2000) proved that there exists a constant $k$ such that $\delta\left(C_{1}, C_{2}\right) \leq k \cdot n^{4 / 3} \log ^{9} n$ for every positive integer $n$ and every $C_{1}, C_{2} \in \mathcal{C}^{n}$, but this gives no concrete information about the upper estimate on Banach-Mazur distance of arbitrary convex bodies of a fixed dimension $n$.

From the first statement of the Theorem we obtain the following Corollary.

Corollary For an arbitrary $C \in \mathcal{C}^{2}$ and a simplex $S$ we have

$$
\delta(C, S) \leq n+2 .
$$

Recall that the author presented a similar proof of this estimate during his talk for the Sixth International Conference on Geometry in Nasholim (Israel) in 1991. This estimate is presented without a proof in the abstract Lassak (1992) of this conference.

For $n=2$ the successive upper estimates 2.5, 2.34 and 2.25 on $\delta(C, S)$ were presented in Lassak (1992); Bálint et al. (1993) and Fleicher et al. (1992). We conjecture that $\delta(C, S) \leq 1+\frac{1}{2} \sqrt{5}(\approx 2.118)$ for every $C \in \mathcal{C}^{2}$ with the equality only for the regular pentagon $P$. For a position of $t(S)$ with respect to $P$ such that $t(S) \subset P \subset\left(1+\frac{1}{2} \sqrt{5}\right) t(S)$ see Fig. 3 in Lassak (1992). Novotný Novotný (1994) proved that for every $C \in \mathcal{C}^{3}$ we have $\delta(C, S) \leq \frac{13}{3}$.

For every $C \in \mathcal{M}^{n}$ we have $\delta(C, S)=n$ (see Grünbaum 1963). This and the Theorem imply the following property: if $S$ is a simplex of maximum volume contained in $C \in \mathcal{M}^{n}$, then the smallest $m$ such that $C \subset m S$ fulfills the inequalities $n \leq m \leq n+2$.

Open Access This article is distributed under the terms of the Creative Commons Attribution Noncommercial License which permits any noncommercial use, distribution, and reproduction in any medium, provided the original author(s) and source are credited. 


\section{References}

Bálint, V., Bálintová, A., Branická, M., Gresák, P., Hrinko, I., Novotný P., Stacho, M.: Translative covering by homothetic copies. Geom. Dedicata. 46, 173-180 (1993). Zbl 0783.52012

Blaschke, W.: Über affine Geometrie III: Eine Minimumeigenschaft der Ellipse. Leipziger Ber. 69, 3-12 (1917). Zbl JFM 46.1112.02

Fleicher, R., Mehlhorn, M., Rote, G., Welzl, E., Yap, C.: Simultaneous inner and outer approximation of shapes. Algoritmica 8, 365-389 (1992). Zbl 0760.68083

Grünbaum, B.: Measures of symmetry for convex sets. 1963 Proc. Sympos. Pure Math., Vol. VII, 233-170, Amer. Math. Soc. Providence, R.I. Zbl 0142.20503

Hudelson, M., Klee V., Larman, D.: Largest $j$-simplices in $d$-cubes: some relatives to the Hadamard maximum determinant problem. Linear Algebra Appl. 241-243, 519-598 (1996). Zbl 0861.15004

John, F.: Extremum problems with inequalities as subsidiary conditions. Courant Anniversary Volume, 187-204 (1948). Zbl 0034.10503

Lassak, M.: On the Banach-Mazur distance between convex bodies. J. Geom. 41, 11-12 (1992)

Lassak, M.: Approximation of convex bodies by triangles. Proc. Amer. Math. Soc. 115, 207-210 (1992). Zbl 0757.52008

Lassak, M.: Packing an $n$-dimensional convex body by $n+1$ homothetical copies. Rev. Roumaine Math. Pures Appl. 51, 43-47 (2006). Zbl 1119.52304

McKinney, J.R.: On maximal simplices insribed in central convex sets. Mathematika 21, 38-44 (1974). Zbl 0288.52008

Novotný, P.: Approximation of convex bodies by simplices. Geom. Dedicata 50, 53-55 (1994). Zbl 0807.52007

Rudelson, M.: Distances between non-symmetric convex bodies and the $M M *$-estimate. Positivity 4, no 2 , 161-178 (2000). Zbl 0959.52008

Szarek, S.J.: Convexity, complexity and high dimensions, Proceedings of the International Congress of Mathemeticians, Madrid, Spain, August 22-30, 2006. Volume II: Invited lectures. Zürich: European Mathematical Society (EMS). 1599-1621 (2006). Zbl 1120.46004

Tomczak-Jaegermann, N.: Banach-Mazur Distances and Finite-Dimensional Operator Ideals. Pitman Monographs and Surveys in Pure and Applied Mathematics 38, Longman Scientifical and Technical, New York (1989). Zbl 0721.4604 